\title{
Effectiveness of Targeted Anticipatory Guidance during Well-child Visits: A Pilot Trial
}

\author{
Namrata A. Magar, MD, Sevdalina Dabova-Missova, MD, and \\ Dwenda K. Gjerdingen, MD, MS
}

Introduction: Anticipatory guidance, an important function of well-child visits, is often brief and not tailored to parents' concerns. This targeted pilot trial evaluated a new method of anticipatory guidance.

Methods: Using an experimental/control study design, we surveyed 137 parents and 31 physicians, comparing their responses to targeted anticipatory guidance (physician-provided education based on parents' concerns) versus usual anticipatory guidance (standard physician lecture on parenting, safety, and nutritional topics).

Results: Overall, physicians appeared less satisfied than parents with the educational component of well-child visits, and they desired changes in the educational aspect of these visits. While physicians believed the targeted approach was easier, control group parents appeared more satisfied with usual anticipatory guidance. The number of anticipatory guidance topics covered in the control group was less than half of that covered in the experimental group.

Conclusions: Our targeted method of anticipatory guidance during well-child visits covered more educational topics and resulted in visits that were easier for physicians, but less satisfactory for parents. Further research is needed to identify methods of anticipatory guidance that are effective and satisfactory for both parents and physicians. (J Am Board Fam Med 2006;19:450-8.)

Well-child visits are used to assess biomedical health, development, behavior, and family functioning, and to provide parent education through age appropriate counseling, referred to as anticipatory guidance. ${ }^{1}$ A recent review of literature on anticipatory guidance during well-child visits reveals that this form of education can have several positive outcomes. For example, anticipatory guidance on mother-infant interaction can improve infant vocal behavior; information about infant temperament can improve parenting skills; education regarding infant sleep patterns is associated with improved sleep; training on "time-outs" can improve parents' use of this method of discipline; information about parents' reading to small children has been associated with improved receptive language development; and education on injury prevention has been related to decreased falls,

This article was externally peer-reviewed.

Submitted 26 October 2005; revised 1 April 2006; accepted 7 April 2006.

From the Department of Family Medicine and Community Health, University of Minnesota, Minneapolis, MN.

Funding: This study was supported by a grant from the American Academy of Family Physicians Foundation.

Conflict of interest: none declared.

Corresponding author: Dwenda Gjerdingen, MD, MS, 580 Rice Street, St. Paul, MN 55103 (E-mail: dgjerdin@ umphysicians.umn.edu). home accidents, and auto passenger injuries. On the other hand, educating parents about child development, television viewing, and firearm storage has not produced any known behavioral benefits. ${ }^{2}$

Despite the potential benefits of anticipatory guidance, many physicians devote little time to this activity, as noted by the results of a recent study showing that infants received an average of 200 seconds of anticipatory guidance, and adolescents 120 seconds per visit. ${ }^{3}$ With such limited time, only a small minority of physicians address all or most important topics, such as car seat or seatbelt use, firearm safety, smoking in the home, healthy weight, physical activity, and diet/nutrition. ${ }^{4}$ Further, the topics that are discussed are usually selected by the physician rather than the parent. ${ }^{5}$ For the clinic population studied here, the well-child visit forms listed 26 to 34 such anticipatory guidance topics, depending on the child's age.

Two national surveys of parents of young children indicate that there are missed opportunities for anticipatory guidance, and parents would like to receive more information about anticipatory guidance topics. ${ }^{6,7}$ Because not all parents value information on the same topics, some experts believe that targeted guidance should be provided on topics of parent concern and patient risk. ${ }^{2}$ Methods 
that can help physicians identify topics for targeted guidance include asking open-ended questions and using pre-visit questionnaires and checklists. ${ }^{2}$

Given the inadequate provision of anticipatory guidance in many practices, the purpose of this study was to compare usual anticipatory guidance (physician-directed verbal education) with targeted anticipatory guidance (based on parents' expressed concerns) on the following outcomes: physician and parent satisfaction, amount of information delivered to parents, perceived learning, and length of visit. We hypothesized that both parents and physicians would be more satisfied with our experimental targeted approach to anticipatory guidance, compared with the standard approach.

\section{Methods}

\section{Population and Study Site}

Bethesda Clinic, the site of this study, is a Family Medicine Residency clinic located near downtown St. Paul, MN, and serves a primarily low-income diverse group of patients. ${ }^{8}$ The clinic is staffed by 24 family medicine residents and 7 faculty.

The study population consisted of physicians and parents of children 0 to 8 years of age who registered for a well-child visit over a 2-month period from November 8, 2004, to January 7, 2005. We selected the 2-month study duration to avoid approaching the same parent more than once, because well-child visits for an individual child seen at this clinic usually occur at intervals of 2 months or greater. Therefore, the vast majority of parents completed only one survey; however, it is possible that a few parents completed more than one. If 2 children from the same family were seen at the same time, we collected only one survey from that parent, and divided the visit time in half.

Physician participants completed more than one survey, and many experienced both experimental and control conditions during the course of the study. We included both English-speaking and non-English-speaking parents for whom an interpreter was available. Only a small minority, if any, of non-English-speaking parents had a physician who spoke their own language. We also included both first-time parents and parents of more than one child. Parents were approached consecutively.

\section{Intervention}

This was a prospective pilot trial, which was approved before its initiation by the University of
Minnesota Institutional Review Board. Bethesda Clinic receptionists were asked to give an informational flyer and consent form to all parents registering their 0 to 8 year old children for well-child visits. Physicians were informed of the study through an educational conference and printed flyer. Parents and physicians who were willing to participate were asked to sign a consent form.

The experimental and control conditions of the study were assigned to each of two equal sides of the clinic (north and south), and this assignment rotated on a weekly basis. Physicians were assigned to these two clinic sides by nursing staff, and these assignments rotated each half-day, so that each physician had approximately equal numbers of exposures to the treatment and control conditions during the course of the study. So, although group assignment was not truly random, we believe that the weekly alteration of treatment conditions together with the twice daily rotation of physician assignments produced a near-random assignment of treatments among physicians and patients.

The intervention consisted of: (1) 2 age-appropriate educational brochures: "Do's and Don'ts for Childcare" and "Your Growing Child," both given to parents just before the physician visit; (2) a Parents' Evaluation of Developmental Status (PEDS) form, also given to parents before the physician visit; and (3) Physician's anticipatory guidance directed toward parents' concerns or questions noted on the "Do's and Don'ts" and PEDS forms.

The "Do's and Don'ts" brochure, created by the authors, consisted of a list of parenting, safety, and nutrition educational directives that were virtually identical to the list of educational topics listed within the "Anticipatory Guidance" section of the clinic's well-child visit forms (see Table 1 for 0 to 5 months "Do's and Don'ts" form). Experimental group parents were asked to circle the items on this brochure that they wanted to discuss with their physician and to indicate that they had read the form by placing a check in a designated spot at the bottom of the form.

The "Your Growing Child" brochure, published by the Minnesota Department of Health, ${ }^{9}$ discussed topics similar to those listed in the "Do's and Don'ts" brochure, but in a more conversational style. The PEDS form ${ }^{10}$ is a checklist of parents' concerns about their child's learning, development, or behavior (see Figure 1 for PEDS Response 
Table 1. Do's and Don'ts for Infant Care: 0 to 5 Months

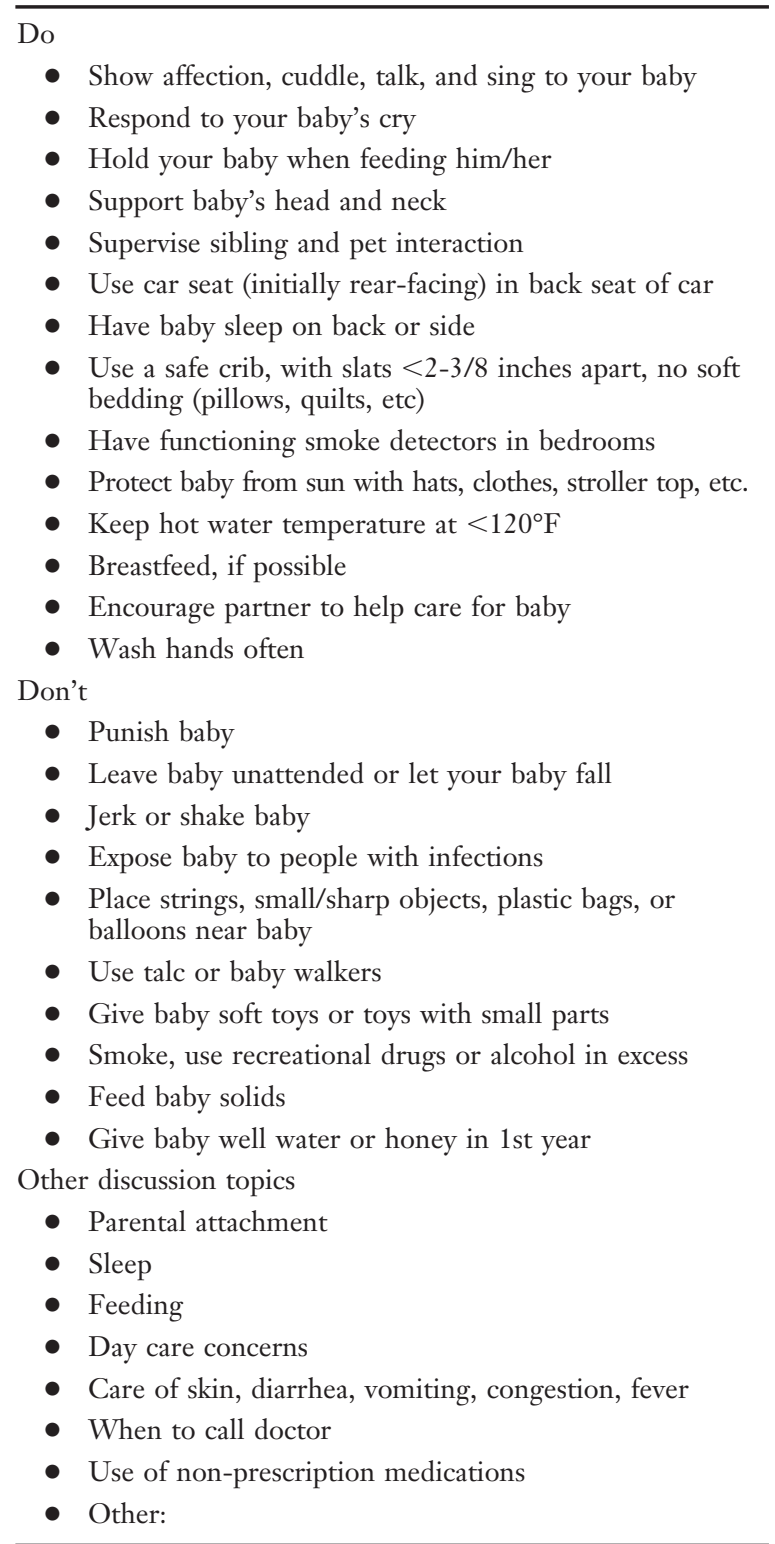

Please check $(\checkmark)$ here _ if you have read the above. If you would like to talk to your doctor about any of the issues listed above, please circle the topic(s).

Form). Experimental group parents were asked to check the items that were of concern to them, before the visit, and the physician's anticipatory guidance was subsequently directed to those items that had been circled or checked on the "Do's and Don'ts" and PEDS forms.

The educational brochures given to experimental group parents were considered to be a substitute for the usual anticipatory guidance lecture, so most experimental group parents did not receive the full lecture. However, parents who had not read the
"Do's and Don'ts" form before the visit were given the usual anticipatory guidance lecture, as presented to control group parents. Experimental group parents were encouraged to read the "Your Growing Child" brochure whenever they were able to do so, either before or after the visit.

Meanwhile, control group parents did not receive the printed materials described above, but instead received usual physician-directed verbal anticipatory guidance, based on the list of 26 to 34 parenting, safety, nutrition, and other age-appropriate educational topics provided under the anticipatory guidance section of the well-child visit form. Given that most physicians do not usually discuss all listed topics, physicians were asked to circle the topics they discussed.

For non-English-speaking parents in either group, an interpreter assisted with the entire visit. In addition, for non-English-speaking experimental group parents, interpreters provided verbal translation for the "Do's and Don'ts" brochure and assistance with completing the PEDS form before the physician visit. Non-English-speaking experimental group parents were also informed that they could obtain a telephone translation of the "Your Growing Child" brochure by calling the appropriate number on the back of the brochure. The presence of an interpreter at a visit was noted on the physician survey.

At the end of each visit, both the parent and physician were asked to complete a brief survey. No data were collected on non-participants.

\section{Surveys}

The parent and physician survey measures were created by the authors for this study, and measured primarily subjective educational and satisfaction outcomes.

\section{The Parent Survey consisted of the following questions.}

Questions for Experimental and Control Group

- How much did you learn about taking care of your child through this visit? (1 to 5 scale, where $1=$ nothing, $3=$ some, and $5=$ a great deal).

- How satisfied were you with the education you received during this visit? ( 1 to 5 scale, where $1=$ very dissatisfied, $3=$ somewhat satisfied, $5=$ very satisfied). 


\section{PEDS RESPONSE FORM}

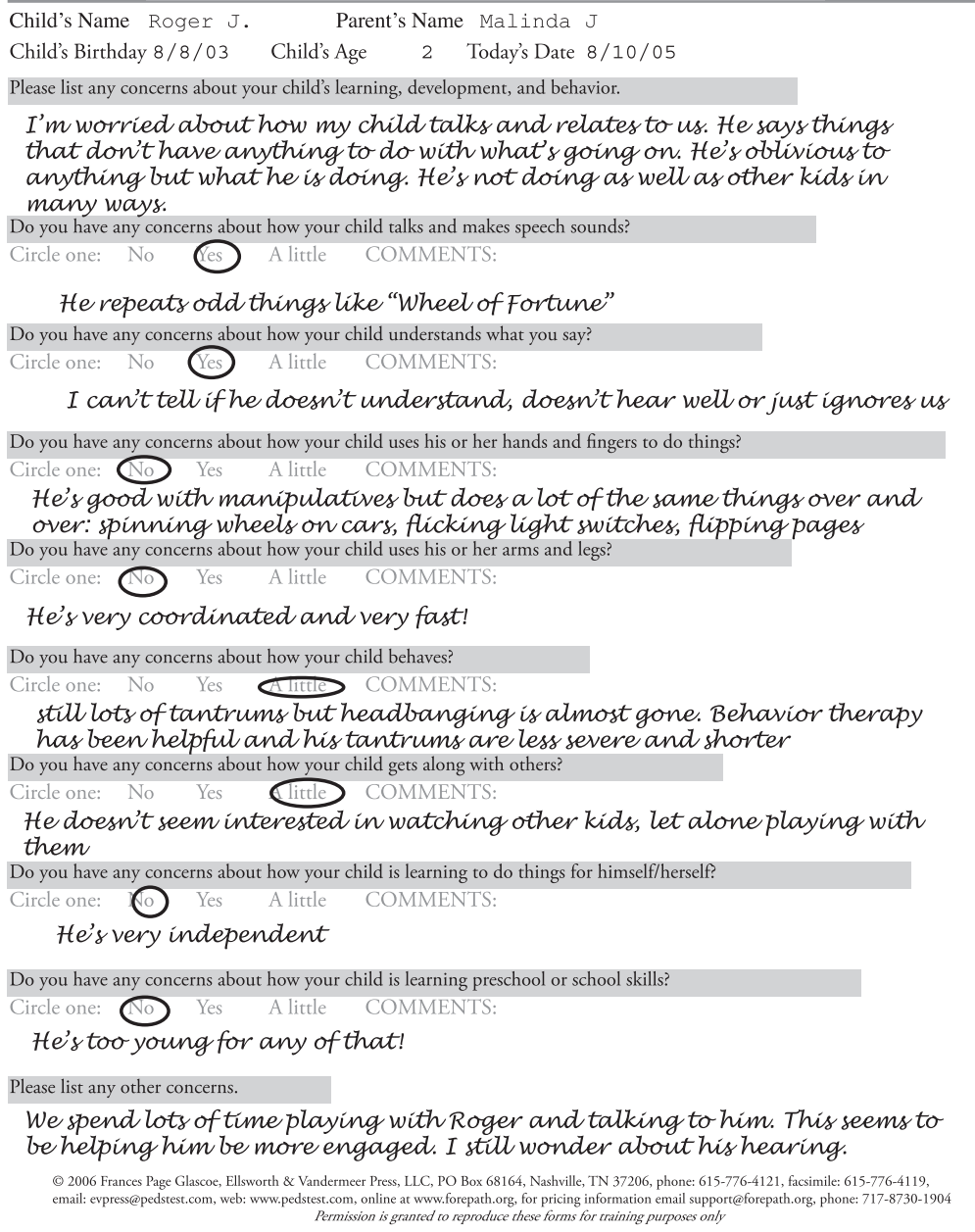

Figure 1. A Parents' Evaluation of Developmental Status (PEDS) response form.

- How well do you think your physician understood your concerns? $(1$ to 5 scale, where $1=$ did not understand, 3 = understood reasonably well, $5=$ understood very well).

- Is there anything you plan to do differently as a result of the education you received during this visit? (yes/no).

\section{Additional Questions for Experimental Group Only}

- Did you complete a PEDS form (asks about your concerns)? (yes/no).

- If yes, how helpful was the PEDS form? (scale of 1 to 5 , where $1=$ not helpful, $3=$ somewhat helpful, and $5=$ very helpful).

\section{The Physician Survey consisted of the following questions}

Questions for Experimental and Control Group

- How satisfied were you with the parent education component of this visit? ( 1 to 5 scale, where $1=$ very dissatisfied, $3=$ somewhat satisfied, $5=$ very satisfied).

- How interested did the parent appear in the educational part of this visit? (1 to 5 scale, where $1=$ very disinterested, $3=$ somewhat interested, 5 = very interested).

- Compared with your average well-child visit before this study, was this visit generally easier or more difficult? ( 1 to 5 scale, where $1=$ much more difficult, $3=$ about the same, $5=$ much easier). 
Table 2. Group Differences in Parents' Responses to Well-Child Visits*

\begin{tabular}{lccc}
\hline Parents' Responses & Experimental Group (n=67) & Control Group (n= 70) & $P$ Value \\
\hline $\begin{array}{l}\text { Perceptions regarding amount learned about } \\
\text { taking care of child through visit }\end{array}$ & $3.89(0.94)$ & $4.20(0.96)$ & .031 \\
$\begin{array}{l}\text { Satisfaction with education during visit } \\
\text { Perceptions about physician's understanding of } \\
\text { his/her concerns }\end{array}$ & $4.17(0.94)$ & $4.51(0.78)$ & .021 \\
$\begin{array}{l}\text { Plans to do something different as a result of } \\
\text { education received (n, \%, } \chi^{2} \text { analysis) }\end{array}$ & $4.52(0.78)$ & $4.64(0.66)$ & $11(15.9 \%)$ \\
\hline
\end{tabular}

* Unless otherwise indicated, responses are given as mean (SD), based on 1 to 5 Likert scale (where 1 is the least desirable and 5 is the most desirable response), and differences were determined by Mann-Whitney-Wilcoxon rank-sum tests.

- Is there anything you would have liked to change about the parent education component of this visit? (yes/no).

- Length of visit: physicians recorded on the survey the time at the beginning and end of each visit.

\section{Additional Questions for Experimental Group Only}

- How many concerns were checked and discussed on the PEDS form?

- Did the parent read the "Do's and Don'ts" form (as indicated by the presence or absence of a check at the bottom of the form)? (yes/no).

\section{Additional Questions for Control Group Only}

- "How many items did you circle or check in the Anticipatory Guidance section of your patient's well-child visit form?"

\section{Post-study Survey}

A small post-study survey was sent to physicians, inquiring about whether they had participated in the study, and if so, the number of experimental and control visits that they had performed.

These surveys did not include demographic data. However, a recent study of parents attending well-child visits at this clinic site showed the following sample characteristics: $90 \%$ female, diverse ethnicity $(65 \%$ Southeast Asian, 15\% African American, 14\% white), 61\% married, 49\% without a high school diploma, 50\% employed, $67 \%$ on public assistance, and a mean age of 25.9 years. ${ }^{8}$

\section{Data Analysis}

We used an "Intent-to-Treat" analysis; therefore, experimental group parents who did not read the educational materials were analyzed together with those who did. Pearson's $\chi^{2}$ was used to compare the two treatment groups on physicians' desires to change anything about the educational component of the visit and parents' plans to do something different as a result of the education they received. Student's $t$ tests were used for the remaining physician comparisons. Because parent distributions were skewed, the Mann-Whitney-Wilcoxon ranksum test was used to test for significant group differences in parents' responses regarding amount learned, satisfaction with education, and physician's understanding. The SPSS program was used to perform all analyses, and a value of $P<.05$ was selected to indicate statistical significance.

Physicians' responses were stratified by parents' English versus non-English-speaking status because it was believed that this variable could significantly impact outcomes such as visit duration or physician's perceived ease/difficulty of the visit. We did not adjust for other confounders because our relatively small sample size limited our power with more complex analyses.

\section{Results}

Parents

There were 137 parent surveys returned, 67 from the experimental group and 70 from the control group. Given that 300 well-child visits for children 0 to 8 years old were performed at this clinic during the 2-month study period, this represents a response rate of $46 \%$. In general, parents from both the experimental and control groups believed they had learned a fair amount during the well-child visit, were satisfied with the educational component of the visit, and believed that their child's physician understood their concerns (Table 2). However, when experimental and control group parents were 
Table 3. Group Differences in Physicians' Responses to Well-Child Visits*

\begin{tabular}{|c|c|c|c|}
\hline Physicians' Responses & Experimental Group $\mathrm{n}=83$ & Control Group n $=105$ & $P$ Value \\
\hline Satisfaction with parent education during visit: & $3.71(1.02)$ & $3.61(0.98)$ & .515 \\
\hline $\begin{array}{l}\text { Perceptions about parent's level of interest in } \\
\text { educational component of visit }\end{array}$ & $3.65(0.96)$ & $3.55(1.10)$ & .544 \\
\hline $\begin{array}{l}\text { Ease/difficulty of visit, compared to average } \\
\text { visit }(1=\text { much more difficult, } 3=\text { about } \\
\text { the same, } 5=\text { much easier })\end{array}$ & $3.69(0.93)$ & $3.19(0.75)$ & .000 \\
\hline $\begin{array}{l}\text { Would liked to have changed educational } \\
\text { component of visit ( }, \%, \chi^{2} \text { analysis) }\end{array}$ & $15(53.6 \%)$ & $18(62.1 \%)$ & .516 \\
\hline
\end{tabular}

* Unless otherwise indicated, responses are given as mean (SD), based on 1 to 5 Likert scale (where 1 is the least desirable and 5 is the most desirable response), and differences were determined by Student's $t$ tests.

compared, parents from the control group believed that they had learned more during the visit, and they felt more satisfied with the educational component of the visit. Despite their relatively high level of satisfaction, only $16 \%$ to $22 \%$ of parents planned to do something different as a result of the anticipatory guidance they received during the visit.

Over $90 \%$ (60/64) of experimental group parents indicated that they had completed the PEDS form, and they generally found the PEDS form to be helpful $($ mean $=3.9$, S.D. $=0.97$ ). Twenty parents who had completed a PEDS form noted at least one concern on the form; the mean number of concerns reported was 2.2 (range $=0$ to $10, \mathrm{SD}=4.0)$.

\section{Physicians}

The 31 participating physicians completed 105 control and 83 experimental group surveys. As noted in Table 3, physicians believed that experimental group visits were easier than control group visits (mean 3.69 vs $3.19, P=.000$ ). This analysis included the $27 \%(18 / 67)$ of experimental group visits where parents did not read the educational materials before the visit. To meet the requirement for anticipatory guidance in these visits, physicians gave their usual educational lecture.

There were no significant experimental versus control group differences in physicians' general satisfaction with the educational component of the visit, or in physicians' perceptions about parents' level of interest in this part of the visit (see Table 3). In addition, visit duration did not differ significantly between groups (mean visit duration for experimental group $=20.9, \mathrm{SD}=10.2$; mean visit duration for control group $=22.2, \mathrm{SD}=8.4 \mathrm{~min}$ utes; $P=.396)$.
Physicians indicated that, for control group visits, the mean and median number of anticipatory guidance items discussed was $10.0(\mathrm{SD}=3.1)$. This represents less than half of the 26 to 34 (number varied with child's age) anticipatory guidance topics listed on the well-child visit forms.

Experimental group visits were shorter than control group visits by 1.3 minutes; however, this difference was not significant. Given the high standard deviation of visit length (8.4 to 10.2), a group difference of 3.8 minutes in visit duration would have been required to achieve a power of 0.80 in this analysis.

Interpreters assisted with 24 visits in the control group, and 9 in the experimental group, for a total of $33(21.6 \%)$ interpreter-assisted visits. For the control group, interpreters' presence, which indicated the non-English-speaking status of parents, was significantly related to decreased physician satisfaction with the educational component of the visit (mean 3.25 vs $3.77, P=.045$ ), and decreased ease of the visit (mean 2.83 vs $3.32, P=.011$ ). In Table 3, when the analyses comparing experimental to control physician responses were stratified by the presence/absence of an interpreter, physicians believed that intervention visits were easier than control visits only for visits that did not require an interpreter (mean $=3.66$ for experimental group visits, 3.32 for control group visits, $P=.028)$. None of the other comparisons in Table 3 (eg, for satisfaction with parent education or perceptions about the parent's level of interest) were significant when stratified by interpreter status.

Physicians gave several subjective responses to the question, "Is there anything you would like to have changed about the parent education component of the visit?" For control group visits, physi- 
cians expressed the following: they wished that these parents had had the opportunity to read the educational brochures given to the experimental group parents $(n=10)$; they felt that there were too many educational topics and too little time to cover them in a visit $(\mathrm{n}=3)$; and they wished that the visits were more focused $(n=2)$ and more interactive $(n=1)$ and that parents would show more interest in anticipatory guidance $(n=1)$. For experimental group visits, physicians wished that interpreters had been more available to translate patient education brochures before the visit or that translated brochures had been available $(\mathrm{n}=6)$. Physicians remarked that parents had not read the educational brochures before the visit because of parents' English illiteracy $(\mathrm{n}=6)$, lack of time $(\mathrm{n}=$ $1)$, and unknown reasons $(\mathrm{n}=3)$.

Fifteen $(48 \%)$ of the physician subjects completed a post-study survey. All but one of the 15 indicated that they had participated in the study, and each physician had participated in an average of 4.9 experimental and 6.8 control visits.

\section{Physicians Compared with Parents}

When physicians were compared with parents on their satisfaction with the educational component of the visit, physicians were significantly less satisfied than parents. These differences remained significant whether the comparisons were made for all visits (mean $=4.34$ for parents, 3.65 for physicians, $P=.000$ ), for experimental visits (mean $=4.17$ for parents, 3.71 for physicians, $P=.006$ ), or control visits (mean $=4.51$ for parents, 3.61 for physicians, $P=.000)$.

\section{Conclusions}

This diverse group of parents was generally satisfied with the anticipatory guidance they received at well-child visits. However, when parents receiving usual anticipatory guidance (a standard physician lecture) were compared with those receiving experimental guidance (written education plus focused verbal guidance directed toward the parent's concerns), "usual" group parents appeared more satisfied and believed they had learned more. These findings were unexpected, given that experimental group parents received a larger quantity of educational material (through printed brochures), and they had more structured opportunities to express their concerns through the PEDS checklist. How- ever, it is entirely possible that this low-income population favored verbal instruction, making the standard lecture approach more desirable. Previous literature on this topic has more commonly addressed the relationship between anticipatory guidance outcomes and physician characteristics, ${ }^{1,11,12}$ rather than parent characteristics. Future research should investigate whether parents' responses to various methods of anticipatory guidance vary with their educational or socioeconomic background.

Despite parents' favorable responses to the education they received during well-child visits, only a minority of parents- $16 \%$ to $22 \%$-planned to change their behaviors in response to the education they received. This disappointing result raises further questions. Did these parents understand and assimilate the advice they received? Did most parents think that they were already giving their child optimal care? If not, did it matter to them that they were not giving optimal care? What does "satisfaction with education" mean to these parents?

Unlike parents, physicians responded more favorably to the experimental anticipatory guidance approach, with the perception that this method was easier than the standard approach. When parents noted no concerns or questions on the "Do's and Don'ts" and PEDS forms, visits were particularly straightforward, because a previsit reading of educational materials by the parents allowed the physician to dispense with the educational lecture. However, this approach did not always work perfectly: $27 \%$ of experimental group parents did not read the "Do's and Don'ts" educational brochure, and some did not complete the PEDS form before the visit. According to physicians' written subjective comments, parents' inattention to these forms before the visit was due to such factors as lack of translated materials for non-English-speaking patients, absence of interpreters before the visit, and having too little time.

Although physicians appeared more satisfied with the experimental than usual educational approach, they were significantly less satisfied than parents with the educational component of the visit. In fact, over half of the physician surveys from both groups indicated that the physician would have liked to change the educational component of the visit. Clues about possible causes of these lessthan-enthusiastic responses are seen in physicians' subjective responses to the question about desired changes in the educational component of the visit. 
They believed there were too many topics to cover in too little time, and they wanted some help with this problem, in the form of printed educational materials, for example. Still, these written materials were not very useful when they were dealing with non-English-speaking parents, and even Englishspeaking parents seemed to prefer the standard educational lecture.

Physicians' approach to the "too many topics/ too little time" dilemma was to curtail the number of anticipatory guidance topics they discussed. Indeed, physicians seeing control group parents discussed fewer than half (10 of 26 to 34) of the topics listed on the well-child visit form. This pattern is consistent with results of a nationally representative sample of 907 primary care pediatricians, who were recently surveyed about how frequently they had discussed various preventive health topics during well-child visits over the previous month. Although over $80 \%$ of the pediatricians discussed one or more recommended preventive health topics during well-child visits, only half of the pediatricians regularly gave anticipatory guidance on more than 2 of 6 topics for children 2 to 5 years of age. ${ }^{4}$ Variables that have been associated with physicians' provision of anticipatory guidance include: the issue's importance, physicians' training and perceived self-efficacy, perceived effectiveness of counseling, presence of office protocol, time, and support staff. ${ }^{1,11}$

The US Preventive Services Task Force has identified 3 categories of behavioral influence on the diffusion of preventive services recommendations into clinical practice: predisposing, enabling, and reinforcing factors. ${ }^{12,13}$ Predisposing factors include knowledge, beliefs and values, attitudes and perceptions, personal health behaviors, confidence, and beliefs about patients' level of interest; enabling factors include competence, reimbursement, organizational characteristics, time, reminder systems, and guidelines; and reinforcing factors include peer support, feedback, evidence of results, and selfefficacy. ${ }^{12,13}$ While this study dealt primarily with predisposing factors (eg, knowledge and attitudes), it is likely that some of the frustration expressed by physician participants was due to variability of certain enabling and reinforcing factors, such as lack of time, parent interest, physician counseling skills, and evidence of results-issues that should be addressed in future studies.
This study has several limitations. First, the results may not be applicable to the general population, because we used a unique patient sample from a single clinic. However, these results may apply to other low-income, ethnically diverse groups. Second, we cannot guarantee the absence of selection bias, because we did not collect data on non-participants; nor can we rule out allocation bias, because we did not use a pure randomization strategy. Third, physician and patient surveys were brief (they did not include demographic data), anonymous, and unlinked, so we cannot evaluate the association of provider or demographic variables with outcomes, and we cannot determine whether there were inherent differences between the 2 treatment groups. Also, given that physicians completed variable numbers of surveys, and a small minority of parents may have completed more than one survey, these observations were not independent.

The study was also limited by our use of subjective outcome measures that had not been previously validated. Future studies on this topic would benefit from validated subjective and objective outcomes, including improvements in parents' behavior and children's health. In addition, we used total visit time as an outcome, when time spent in anticipatory guidance may have been more specific to the purposes of this study. The inclusion of nonEnglish-speaking patients, although theoretically a strength, was somewhat problematic here, because we did not have translated printed educational materials for the non-English-speaking Hmong, Hispanic, Somali, and other ethnic groups. It should be noted, however, that translated materials would not have been useful to many of these parents, particularly the Hmong (our largest non-English-speaking subgroup), as most of the non-English-speaking Hmong are not literate in any language.

However, the study also has some important strengths. We used an experimentally designed research trial with intent-to-treat analysis to identify a method that may help make well-child visits less cumbersome for physicians, provided that appropriate resources- eg, translated brochures and previsit interpreters for non-English-speaking parents-are available. Importantly, we found that physicians did not look with favor on "usual" anticipatory guidance. Further research is needed, to identify methods for improving parental education on child health, development, and safety in a way that is satisfactory and efficacious for both parents 
and physicians. It would also be important to determine whether optimal methods of anticipatory guidance vary with parents' ethnicity, other demographic characteristics, and the child's age and position in the family. Furthermore, although it is important to look at the knowledge component of prevention in practice, we also need to address the enabling and reinforcing factors that allow our anticipatory guidance to take root in the lives of parents and children.

In conclusion, the patients studied here were generally more satisfied with the educational component of well-child visits than were the physicians, most of whom desired changes in this component of well-child visits. Yet, even though parents were generally satisfied with the anticipatory guidance they received, only a small minority planned to change their behaviors. The educational intervention tested here-a focused method of anticipatory guidance-was seen as an improvement by physicians, but not by parents. Additional research is needed to identify methods of anticipatory guidance that are satisfactory for both parents and physicians, and that produce positive behavioral change.

We acknowledge Christine Danner, $\mathrm{PhD}$, for contributions to survey development. NAM and SD-M participated in the study design, data collection, and preparation of the manuscript; DKG participated in the study design, data collection and analysis, and preparation of the manuscript.

\section{References}

1. Dinkevich E, Ozuah PO. Well-child care: effectiveness of current recommendation. Clin Pediatr 2002; 41:211-7.

2. Nelson CS, Wissow LS, Cheng TL. Effectiveness of anticipatory guidance: recent developments. Curr Opin Pediatr 2003;15:630-5.

3. Norkin Goldstein EN, Dworkin PH, Bernstein B. Time devoted to anticipatory guidance during child health supervision visits: how are we doing? Amb Child Health 1999;5:113-20.

4. Galuska DA, Fulton JE, Powell KE, et al. Pediatrician counseling about preventive health topics: results from the Physicians' Practices Survey, 19981999. Pediatrics 2002;109:E83-3.

5. Cheng TL, DeWitt TG, Savageau JA, O'Connor KG. Determinants of counseling in primary care pediatric practice: physician attitudes about time, money, and health issues. Arch Pediatr Adolesc Med 1999;153:629-35.

6. Halfon N, Olson L, Inkelas M, et al. Summary statistics from the National Survey of Early Childhood Health, 2000. Vital Health Statistics 15(3). National Center for Health Statistics, 2002.

7. Schuster MA, Duan N, Regalado M, Klein DJ. Anticipatory guidance: what information do parents receive? What information do they want? Arch Pediatr Adolesc Med 2000;154:1191-8.

8. Jhanjee I, Saxeena D, Arora J, Gjerdingen D. Parents' health and demographic characteristics predict noncompliance with well-child visits. J Am Board Fam Pract 2004;17:324-31.

9. Minnesota Department of Health, "Your Growing Child" brochures for newborns, 1-3 months, 6-8 months, 9-11 months, 12-14 months, 15-17 months, 18-23 months, 2 years, 3 years, 4-6 years, 7-10 years: Available from: http://www.dhs.state. mn.us/main/groups/business_partners/documents/ pub/dhs_id 026299.hcsp

10. Glascoe FP. Collaborating with parents: using parents' evaluation of developmental status to detect and address developmental and behavioral problems. Nashville (TN): Ellsworth \& Vandermeer Press LLC, 1998-2002.

11. Erickson MJ, Hill TD, Siegel RM. Barriers to domestic violence screening in the pediatric setting. Pediatrics 2001;108:98-102.

12. Lawrence RS. Diffusion of task force recommendations: diffusion of the U.S. Preventive Services Task Force recommendations in practice. J Gen Intern Med 1990;5(Suppl):S99-103.

13. Green LW, Eriksen MP, Schor EL. Preventive practices by physicians: behavioral determinants and potential interventions. Am J Prev Med 1988;4:101-7. 